\title{
Antropología
}

\section{Las categorías de género vistas por los jóvenes universitarios de la Facultad de Enfermería de la Universidad de Cádiz}

\section{Gender categories seen by university students in the School of Nursing at the University of Cádiz}

\section{As categorias de gênero visto por estudantes universitários da Escola de Enfermagem da Universidade de Cádiz \\ ${ }^{1}$ Lazaro González Calvo, ${ }^{2}$ Alvaro Bernalte Benazet \\ ${ }^{1}$ Enfermero y Ldo. Antropólogía social y cultural. Mozambique. \\ ${ }^{2}$ Antropólogo Social y Cultural, Dr. en Medicina. Universidad de Cádiz (UCA), Facultad de Enfermería. Algeciras.}

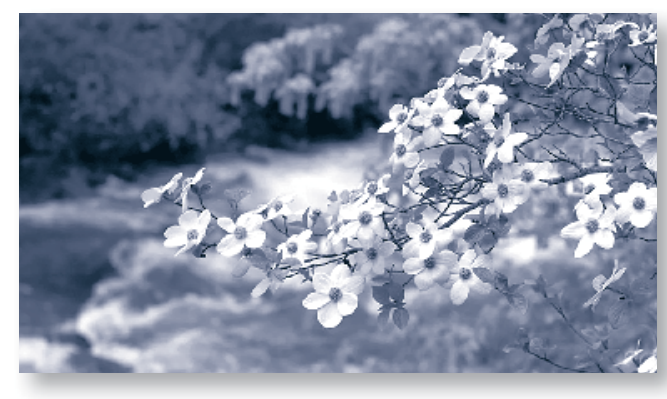

Gender categories seen by university students in the School of Nursing at the University of Cádiz

\section{ABSTRACT}

Objective. Our aim has been closer to the vision of young people about the current meanings of masculine and feminine, defined as objects of study to final year university nursing.

Methodology. Our position is qualitative ontologically and epistemologically constructivist, using techniques: participant observation, the nominal group technique and focus group techniques.
Results. The results are that the current female profile is based on the following terms: independent, feisty, single, self, and emotional and men as follows: honest, hardworking, strong, sensitive, and tolerant.

Conclusions. Among the findings could point out that men and women who seemed to be simply human beings in their conversations, do not agree on any ITEM descriptive of the masculine and feminine words are different than they were previously mentioned stereotypes, but still do not agree no point profiles.

Keywords: women's perception, perception of male.

As categorias de gênero visto por estudantes universitários da Escola de Enfermagem da Universidade de Cádiz

\section{RESUMO}

Objetivo. Nosso objetivo tem sido mais próximo da visão dos jovens sobre o signifi- 
cado atual do masculino e feminino, definidos como objetos de estudo para a enfermagem do último ano de universidade.

Metodologia. Nossa posição é de natureza qualitativa ontológicamente, e epistemologicamente construtivista, utilizando técnicas: observação participante, a técnica de grupo nominal e as técnicas de grupo focal.

Resultados. Os resultados são que o perfil atual do sexo feminino baseia-se nos seguintes termos: independente, resoluto, único, e emocionais e os homens como se segue: honesto, trabalhador, forte, sensível e tolerante.

Conclusões. Entre os resultados poderiam indicar que homens e mulheres que pareciam estar simplesmente seres humanos em suas conversas, não concordar com qualquer elemento descritivo de masculino e feminino são diferentes do que eram anteriormente mencionado estereótipos, mas ainda não correspondem os perfis em qualquer ponto.

Palavras-chave: percepção das mulheres, a percepção do sexo masculino.

\section{RESUMEN}

Objetivo. Nuestro objetivo ha sido acercarnos a la visión de los jóvenes sobre los significados actuales de lo masculino y lo femenino, definiendo como sujetos de estudio a los universitarios de último curso de Enfermería.

Metodología. Ontológicamente nuestra posición es cualitativa, y epistemológicamente constructivista, utilizando como técnicas: la observación participante, la técnica de grupo nominal y la técnica de grupo focal.

Resultados. Los resultados son que el perfil femenino actual está basado en los siguientes términos: independiente, luchadora, única, autosuficiente, y sentimental y el masculino de la siguiente forma: honrado, trabajador, fuerte, sensible, y tolerante. Conclusiones. Entre las conclusiones podríamos destacar que hombres y mujeres que parecían ser simplemente seres humanos en sus conversaciones, no coinciden en ningún ITEM descriptivo de lo masculino y lo femenino, es decir son diferentes a lo que eran antes los estereotipos mencionados, pero siguen sin coincidir en ningún punto los perfiles.

Palabras clave: percepción de femenino; percepción de masculino.

\section{INTRODUCCIÓN}

Para nosotros la preocupación por los significados había sido estimulada por un desarrollo conceptual de Geerz en cuanto a la cultura, ya que este explicaba la existencia de dos tipos de información necesarios para la vida de los humanos, la una que nos llega con los genes y la otra con la cultura.

Entre las aportaciones, es decir aquello que nos es trasmitido mediante nuestra enculturación, las categorizaciones (divisiones al fin y al cabo) son una buena muestra de ello, por ejemplo la debida al sexo/género, que implican de forma importante nuestro futuro como seres humanos.

Todas las sociedades buscan dotarse de valores y criterios para garantizar su reproducción, tomar decisiones, organizar la producción y regular sus intercambios. En esta tarea se establecen una serie de roles y clasificaciones que permiten su funcionamiento. Una de las clasificaciones determinantes en la organización social de todos los pueblos ha sido la "división" entre lo femenino y lo masculino, que ha tomado como base las diferencias sexuales entre hombres y mujeres. La mayoría de las culturas construyeron sobre esas distinciones biológicas, un conjunto de atributos, 
roles, percepciones, prescripciones, derechos y obligaciones, elementos estos, que acabaron siendo percibidos como naturales, generando una confusión entre sexo (lo biológico) y género (lo social), como comenta Miano, $\mathrm{M}$. (2002:25): "El género es una dimensión fundamental de las relaciones sociales que en nuestra sociedad expresa, junto con las diferencias de clase o estratificación social y étnicas, otra dimensión de la desigualdad social"

El concepto sexo hace referencia a las condiciones orgánicas que establecen las diferencias biológicas entre hombres y mujeres, condiciones congénitas y universales. El concepto género es fundamental para entender su lugar estructurante en las relaciones sociales (principio de ordenamiento jerárquico de la práctica social) y para su análisis se requiere establecer: primero, la relación entre sexo y género y segundo, los efectos de tal distinción.

El concepto de género, incluye además, las características socioculturales e históricas que determinan la forma de interacción entre hombres y mujeres, la división de funciones y las características modificables en el tiempo y que varían ampliamente de una cultura a otra. Bajo esta acepción, género es una categoría social que permite analizar las responsabilidades, limitaciones, papeles y oportunidades diferentes, para hombres y mujeres en el interior de su unidad familiar, comunidad, escuela, cultura y unidad de producción, entre otras, en tanto que producción social se presenta a los individuos como una realidad objetiva y subjetiva, recreada por ellos "los actores sociales", con base en los significados proporcionados por la historia, la cultura y el lenguaje.

Esta temática (vigente: como síntoma social), así como por nuestra preocupación como antropólogos y sanitarios, ante la realidad social que nos acontece, donde se desdibujan estas prescripciones (discurso cultural: sobre lo masculino y femenino), deconstrucción del género, dando paso a nuevas resignificaciones, sobre todo en los jóvenes, quienes reconstruyen el discurso (lo definen), que les servirá como "guión o papel" en la sociedad, siendo este proceso (nuestro objeto de atención), en definitiva lo que nos ha conducido, hacia nuestra perspectiva investigadora, focalizando en lo que definimos como sistema: "sexo/género".

Por ello la selección de los sujetos de estudio han sido jóvenes, que eran los alumnos con mayor trayectoria universitaria, de la actual Facultad de Enfermería de la Universidad de Cádiz, es decir los alumnos de último año de carrera.

La revisión sobre dicha temática es compleja, por el ingente material y el número de autores que han abordado la cuestión, la revisión inicial de manuales, que hubieran tocado con cierta profundidad el sistema de género, e insistimos prioritariamente desde la Antropología; y nuestra segunda fase fue la revisión de artículos de interés.

Obviamente, pues, nuestra aproximación a la bibliografía viene dada por el objeto que nos preocupaba, que no es otro que: "el significado que los jóvenes dan a las categorías culturales denominadas género".

Debemos mencionar que existe una literatura amplia sobre feminismo, o lo femenino, no así sobre lo masculino, ello es debido al trabajo de las autoras de las tres oleadas feministas, pero que implica a veces una confusión entre género y femenino.

Estas categorías no han sido dos en todas las culturas, sabemos de la existencia en las tribus norteamericanas de los Berdaches de la tribu Crown, y en la actualidad- de los muxe' en el México Zapoteca; una concepción que nos puede parecer más moderna que las que 
oímos a nuestro alrededor, crear posibilidades para que las personas se identifiquen con categorías que responden a sus formas de entender la vida social y el mundo, y que les permiten un desarrollo en la sociedad y no una estigmatización.

Cómo nos vamos a aproximar una realidad social a estudiar, depende en gran medida de nuestra concepción de la realidad, de su naturaleza. Para nosotros esta es cognoscible en la vida cotidiana de los actores sociales, pues son ellos quienes crean esa realidad, conocen "el porqué" de sus acciones, y son capaces de trasmitirlo a los investigadores; por tanto nuestra postura es cualitativa, pero no antipositivista, pues como Bericat, creemos que la utilización de otro tipo de técnicas nos ayuda a ir, a veces más lejos en nuestras investigaciones.

\section{MARCO TEÓRICO}

Nuestro marco teórico, podemos definirlo en cuanto a la ontología como una mirada hermeneútica cuyo foco central de análisis, comprende la perspectiva de los actores observados, es decir los jóvenes alumnos de Enfermería; epistemológicamente adoptaremos como perspectiva la contructivista que mantiene una posición relativista de la realidad social, realidad que se construye sobre la base de las interacciones en la vida cotidiana, y que entiende que los sujetos conocen y pueden explicar sus actos y motivaciones; y metodológicamente nos situaremos en el campo de la etnografía, por tanto utilizaremos una metodología basada en el trabajo de campo, en el estar en contacto con los jóvenes y utilizar la observación participante, entrevistas, y algunas técnicas de grupo focales.

Pero nos gusta pensar como opinan Coffey; Atkinson (2005:17) "La búsqueda del método perfecto de análisis de datos es in- fructuosa" y tener la certeza que no tenemos la única manera correcta de analizar nuestra información, tal como establecen los mismos autores, así partiendo de un cierto eclecticismo, solo hay que buscar cuál es la más interesante para nuestros datos "No hay una sola manera correcta de analizar los datos cualitativos; además, es esencial hallar modos de usar los datos para pensar con ellos. Tenemos que encontrar los modos más productivos de organizar e inspeccionar nuestros materiales." (2005:17).

\section{MÉTODO}

Las actividades previas fueron: la solicitud de permiso para realizar este estudio en la Escuela Universitaria de Enfermería de Algeciras de la Universidad de Cádiz; la definición del rol de los investigadores como investigadores sociales inexpertos; definir las fechas y el cronograma para las actividades, y solicitar alumnos voluntarios para participar en las técnicas

Definimos nuestras unidades de observación y análisis de la siguiente forma: nuestra unidad de observación serían los alumnos con mayor trayectoria universitaria de la Escuela de Enfermería de Algeciras, es decir los alumnos de último año de carrera; y nuestras unidades de análisis: la categoría femenino, la categoría masculino, cruzadas por el género de los informantes, y nuestra observación participante como coordinadores y analistas de las técnicas.

En está etnografía utilizamos en el trabajo de campo: la observación participante, la técnica de grupo nominal (TGN) y la técnica de grupos focales (TGF). Las TGN tenían como objetivo que alumnos y alumnas definieran terminológicamente, lo que ellos y ellas entendían como términos que perfilaban actualmente lo masculino y lo femenino respectivamente, y la importancia de cada uno de ellos; 
los grupos focales posteriores nos permitieron establecer claramente el significado de los términos obtenidos, también de forma diferenciada por género.

A continuación la TGN conjunta revisó el trabajo de los grupos anteriores, volviendo a valorar, conjuntamente, ambos perfiles; realizándose una TGF, donde se definieron los términos de estos dos perfiles.

El Universo a estudiar eran los alumnos de 3er año de la Escuela Universitaria de Enfermería de Algeciras de la Universidad de Cádiz; las muestras escogidas fueron tres para adaptarse a la dimensión que permitían las propias técnicas, y su elección al azar por su intencionalidad de participar hasta obtener el número deseado: así las alumnas que se presentaron voluntarias para realizar unas técnicas grupales con ellas; los alumnos que se presentaron voluntarios para realizar unas técnicas grupales con ellos; y las alumnas y alumnos, que entre los anteriores se presentaron voluntarios para las últimas dos técnicas conjuntas.

\section{RESULTADOS}

La TGN con alumnas definió un perfil de lo femenino basado en los siguientes Items: independiente, luchadora, única, liberal, autosuficiente y persona, por este orden de importancia (Tabla $n^{\circ} 1$ ); la TGN con alumnos definió lo masculino con los siguientes términos: honrado, trabajador, leal, fuerte, sensible y tolerante (Tabla $\left.n^{\circ} 2\right)$.

Las TGFs con alumnos o alumnas definieron los términos aportados por las anteriores técnicas, así las alumnas definían: independiente con "no depender de nadie” y matizándose en la "no dependencia económica" yla "no dependencia emocional"; el término luchadora, en que las mujeres habían sido luchadoras por definición y por lo tanto como obligación

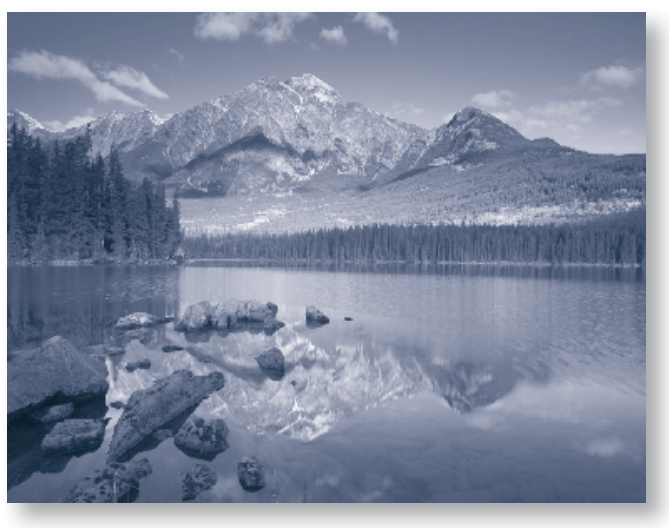

cultural y social; pero que hoy día se entendía por mujer luchadora "la que lucha en pos de sus objetivos personales"; por única, aunque inicialmente no había acuerdo, y el consenso se establecía con respecto a este término, tardó en llegar, la consideración de "única no como miembro de una categoría (hembra, mujer, mari,...) sino como un ser absolutamente individual y diferente a los / las demás", lo cual implicaba o reforzaba como pensamiento la salud mental; por liberal, reconocemos que este término produjo un cierto revuelo, la discusión fue más generalizada, pero se llegó a un acuerdo, considerando que se refería a "liberal como una actitud de tolerancia" , y "como una situación de poder elegir"; el concepto de autosuficiente, hizo al grupo divagar, a pesar de ser reconducido varias veces por el moderador, la autosuficiencia "parecía romper con el estatus y los roles de la mujer tradicional" y planteaba a una mujer "cuyo valor se debía a sí misma”, y que "necesitaba menos de los demás"; por último sobre persona, hubo mucha controversia, pero quizás el punto de encuentro fue "no ser encasillada en una categoría" "evitar la cosificación (objeto) que imprimen ciertas categorías como las de género".

Los alumnos tipificaron lo masculino en esta TGN (Tablas $n^{\circ} 3$ y no 4 ) de la siguiente forma: honrado, la idea que llevo a todos a 
una aceptación del término tal como se debía entender hoy día, era: "un hombre con una forma de ser confiable" es decir que "se puede confiar en él y él tiene confianza en los demás"; trabajador, fueron aproximándose al concepto, hablando de: "perseverancia'"' siempre intentar estar al día" (aquí se ve una influencia del entorno competitivo en que se desarrolla la vida de los profesionales de la salud), "no deja nada a medias", etcétera; Así el hombre trabajador es "alguien que se esfuerza por subir y mejorar su estatus laboral y vital".; leal, claro que lo traducían casi todos por "fiel”, pero la descripción más apoyada fue "un hombre que siempre está ahí, de una forma humilde y veraz"; fuerte, se circunscribió a la exposición de dos categorías de fortaleza, a saber: "fortaleza física" y "fortaleza psíquica”, después de un breve ronda para ponerse de acuerdo, todos expresaron que lo que más definía lo que ellos pensaban era la "fortaleza psíquica"; sensible, dio origen a una discusión mayor de lo que cabría esperar por dos tomas de posición de otros tantos participantes en el sentido "que el hombre se ve abocado por presión social a ser sensible y que se ha pasado" y la otra "que se está afeminando / sensibilizando el hombre sin encontrar el punto", amén de algunas aportaciones en ambos sentidos, se llegó a un acuerdo sobre lo que implicaba para ese hombre de hoy: "el hombre debe ser sensible porque es parte de su forma de ser como ser humano, y no puede permitir que el 'estereotipo hombre macho' sea el que le obligue a no ser sensible; y tolerante, cuyo consenso se obtuvo sobre dos aspectos rápidamente, quizás debido a la hora, "abierto" y "capaz de cambiar de opinión”, así ese hombre de hoy tolerante debía "ser una persona abierta de ideas, capaz de compartirlas e intercambiarlas y también a cambiar su opinión”.

El trabajo conjunto provocó situaciones de

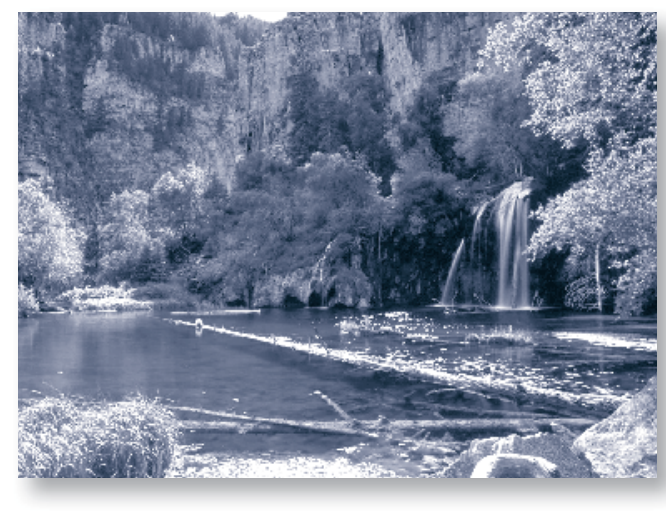

tensión entre alumnas y alumnos, y también dentro de los alumnos y de las alumnas, de hecho la valoración del perfil cambia y desaparecen dos términos, en el femenino liberal y en el masculino el de leal (Tabla no ${ }^{\circ}$ ).

De esta forma la descripción del perfil femenino, quedó así: independiente, como no dependiente de nadie ni a nivel económico, ni emocional; luchadora, por sus objetivos personales; única, como un ser absolutamente individual y diferente a los / las demás; autosuficiente, que necesitaba menos de los demás y cuyo "valor" se debía a si misma, a diferencia de la mujer tradicional dependiente y reconocida por aquel rol y sentimental, actúan y reaccionan emotivamente; y el perfil masculino: honrado, una persona en la que se puede confiar y con confianza hacia los demás; trabajador, alguien que se esfuerza por subir y mejorar su estatus laboral y vital; fuerte, alguien con fortaleza sicológica; sensible, como es parte de su forma de ser como ser humano, y no puede permitir que el 'estereotipo hombre macho' sea el que le obligue a no ser sensible; y tolerante, una persona abierta de ideas, capaz de compartirlas e intercambiarlas y también de cambiar su opinión.

Hay que mencionar que las definiciones realizadas y consensuadas, se han acercado mucho a las etimológicas como podemos observar en las Tablas $n^{\circ} 6$ y n $^{\circ} 7$. 


\section{CONCLUSIONES}

\section{Procedentes de la observación participante:}

1. Las alumnas, que si bien iniciaron sus aportaciones, con muchas categorías que responden a un estereotipo de la mujer clásico, podíamos decir, primero se deshicieron de los ítems más "light", pero ya en el segundo cribage desaparecieron quizás los aspectos más clásicos en nuestra cultura, como pueden ser: familiar, maternal, cuidadora y sensible.

2. Los alumnos, al principio como sucede a veces con los informantes iniciaron el proceso introduciendo algún ítem, que suponemos pensaban que queríamos oir, por ejemplo "femenino"; pero en la segunda fase de la técnica, creemos que se centraron y desaparecieron los que habían superado "curiosamente" la primera fase, por ejemplo igualitario y no agresivo.

\section{Procedentes de las técnicas de grupo nominal iniciales:}

3. Lo que podríamos destacar es que hombres y mujeres que parecían ser simplemente seres humanos en sus conversaciones, no coinciden en ningún ITEM descriptivo de lo masculino y lo femenino, es decir son diferentes a lo que eran antes los estereotipos mencionados, pero siguen sin coincidir en ningún punto los perfiles

\section{Procedentes de los grupos focales:}

4. A pesar de que dentro de los conceptos tuvieron que consensuar diferentes posturas, si tienen cierto parecido semántico con el Diccionario de la Lengua Española.

6. En las alumnas la búsqueda de la definición, clarifica algo la descripción de lo que ellas consideran lo femenino, que sugiere una búsqueda de libertad que no han tenido -en general- las mujeres por el hecho de serlo de ahí el abandono de las categorías que encorsetan, amén de la defensa de lo propio, sean objetivos o criterios.

7. En los alumnos, existe una cierta complicidad de grupo, de hecho algunas categorías que se reivindican se hacen desde la acusación de "hurto" no de "dejadez", asimismo son tolerantes, pero enfatizan que tienen "derecho" a cambiar de opinión, si aceptamos las opiniones de los demás obviamente que se puede cambiar de opinión, por qué matizarlo, es una "maniobra de defensa", por último reivindican quizás aquello en lo que las mujeres parecen más fuertes, el aspecto mental.

\section{Procedentes de las TGF conjuntas:}

8. Ante la presión de las alumnas el término lealtad de los alumnos se descuelga definitivamente, con la presión nos referimos simplemente a preguntar en el momento que se corresponde con las aclaraciones sobre las categorías en la técnica, por ciertos ítems y la respuesta no verbal ante las explicaciones de los alumnos, que podríamos definirlo llanamente como incredulidad.

9. Los chicos concretan una victoria parcial de su esquema de lo femenino, introduciendo el concepto sentimental, y lo concretan expresivamente de forma no verbal.

10. La consideración de que el grupo ha consensuado más las categorías, por la manifiesta concentración de votos y por los cambios en las posiciones.

\section{Procedentes de los perfiles:}

11. Obviamente hay una coincidencia en los dos grupos de querer cambiar sus perfiles o un desacuerdo con el perfil de lo masculino y lo femenino que se ha dado en nuestra cultura clásicamente. 
12. El grupo masculino, sin embargo, si pretende que la mujer mantenga algo del perfil anterior, como la categoría sentimental.

13. Por último decir que el grupo femenino que participó con los alumnos en algún momento pareció o pretendió que estos se disculparán de la actitudes que hacia el género femenino se habían tenido.

\section{BIBLIOGRAFÍA}

- Berger, P.; Luckmamn, T., (1988) "La construcción social de la realidad", Biblioteca de Sociología, Amorrortu, Buenos Aires. (pp. 17-24, 26-35; y 37-44)

- Barley, N., (1995) “El antropólogo inocente”, Anagrama, Barcelona.(pp. 20-22: y 156-159)

- Coffey, A.; Atkinson, P. (2005). "Encontrar el sentido a los datos cualitativos. Estrategias complementarias de investigación”. Publicaciones de la Universidad de Alicante. Alicante. (pp. 16-31)

- Connell, Robert. W. (1987) a "Gender and Power: Society, the Person and Sexual Politics, (parte II), “The Structure of gender Relations”, pags. 89-164 y el capítulo 6, “Gender Regimes and the Gender Order", pags. 119-142, Standford, Carolina, Standford University Press.

- . Coffey, A.; Atkinson, P. (2005). "Encontrar el sentido a los datos cualitativos. Estrategias complementarias de investigación". Publicaciones de la universidad de Alicante. Alicante. (pp. 16-21).

- Delphi, Christine. (1991) "Penser le genre, quells problemas? Sexe et genre. De la hiérarchie entre les sexes. CNRS; París.

- Dey, I. (1993). “Qualitative data analysis: a user friendly guide for social scientists" Londres. Routledge and Keagan Paul. (pp. 47-49)

- De Beauvoir, S. (1999) “El Segundo sexo, Volumen I, Los hechos y los mitos. Cátedra. Madrid.(pag. XXIII)

- De Lauretis, T. (1991) "Queer theory: lesbian and gays sexualites: An Introduction. and Differences 3 (2).

- Delbecq, L.; Van de Ven, A.; Gustafson, D. (1989). “Técnicas grupales de planeación”. México. Ed. Trillas.

- Gil Calvo, E., (1997) “El nuevo sexo débil”, ed. Temas de
Hoy, S.A. (pp. 14-31)

- Guasch, O., (1997) "La observación participante", (Cuadernos metodológicos n 20), CIS, Madrid. (pp. 5-24)

- Harris, M., (1998) “Introducción a la Antropología General”, Alianza Editorial, Madrid. (pp. 564-571)

- Heritier, F. (2002), “Masculino. Femenino”. Ed. Ariel (pp.4-62)

- Huberman, A. M; Miles, M. B. (1994). Data management and analysis methods. En: Denzin, N. K., Lincoln, Y. S. (eds). Handbook of qualitative research. Sage. Tousand Oaks (California). (pp. 428-444).

- Langaney, A, (1985). “Sexo e innovación”. Ediciones Juan Granica S. A. Madrid (pp.147-187)

- Lomas, C., (1999) “iIguales o diferentes? (Género, diferencia sexual, lenguaje y educación)”. Editorial Paidos, Barcelona.

- Mead, M. (2006). “Sexo y temperamento”. Ediciones Paidos. Barcelona. (pp. 23-86)

- Miano, M. (2002) "Hombre mujer y muxe' en el Istmo de Tehuantepec”. México. INAH. (pag. 22-25, 65-72)

- Murano, R. M. (2004) “Femenino y Masculino”. Editorial Trotta.

- Narotzki, S. (1995) “Mujer, mujeres y género". CSIC. Madrid. (pp. 11-16; y 32-36).

- Philipp, R. (2001) “Cuestiones Actuales de Sociología del Género". Centro de Investigaciones Sociológicas. Madrid.

- Rubin, Gayle. (1986) “El tráfico de mujeres: notas sobre la economía política del sexo”. Nueva Antropología. Vol. 8, núm. 30. Barcelona.

- Soley-Beltrán, P. (2009). “Transexualidad y la matriz heterosexual”. Ediciones Bellaterra. Barcelona. (pp. 27-41; y 103-114).

- Valcuende, J. M.; López, J. (eds) (2003). "Hombres. La construcción cultural de las masculinidades". Talasa Editores. Madrid. (pp. 9-21; 25-51; y 53-65)

- Wittig, M., (1979) “No se nace mujer” (One is not born a Woman) Feminist Issues. 1,2 1979.

- Wolcott, H. (1994). “Transforming up qualitative data: description, analysis, and interpretation. Tousand Oaks. Sage. (pag. 36-47). 
Tabla $n=1$. Resultados de la primera TGN con alumnas, describiendo los términos que representan lo femenino actualmente y la importancia que ellas le dan

\begin{tabular}{|c|c|c|}
\hline ITEM & TÉRMINO UTILIZADO & $\begin{array}{c}\text { PUNTUACIÓN } \\
\text { OBTENIDA }\end{array}$ \\
\hline 2 & INDEPENDIENTE & 320 \\
\hline 5 & LUCHADORA & 280 \\
\hline 21 & ÚNICA & 240 \\
\hline 6 & LIBERAL & 220 \\
\hline 9 & AUTOSUFICIENTE & 220 \\
\hline 1 & PERSONA & 200 \\
\hline
\end{tabular}

Elaboración propia

Tabla no 2. Resultados de la primera TGN con alumnos, describiendo los términos que representan lo masculino actualmente y la importancia que ellos le dan

\begin{tabular}{|c|c|c|}
\hline ITEM & TÉRMINO UTILIZADO & $\begin{array}{c}\text { PUNTUACIÓN } \\
\text { OBTENIDA }\end{array}$ \\
\hline 1 & HONRADO & 540 \\
\hline 5 & TRABAJADOR & 380 \\
\hline 21 & LEAL & 260 \\
\hline 6 & FUERTE & 260 \\
\hline 9 & SENSIBLE & 160 \\
\hline 1 & TOLERANTE & 120 \\
\hline
\end{tabular}

Elaboración propia

Tabla $n=3$. Resultados de la segunda TGN con alumnas y alumnos, sobre lo femenino y su importancia comparada con la que se le había otorgado en la 1a TGN

\begin{tabular}{|c|c|c}
\hline $\begin{array}{c}\text { ORDEN SEGÚN LA } \\
\text { PUNTUACIÓN TGN } \\
\text { ANTERIOR }\end{array}$ & $\begin{array}{c}\text { ORDEN SEGÚN LA } \\
\text { TÉRMINO UTILIZADO }\end{array}$ & $\begin{array}{c}\text { IMPORTANCIA EN ESTA } \\
\text { TGN }\end{array}$ \\
\hline $1^{\circ}$ & INDEPENDIENTE & $2^{\circ}$ \\
\hline $2^{\circ}$ & LUCHADORA & $3^{\circ}$ \\
\hline $3^{\circ}$ & ÚNICA & $4^{\circ}$ \\
\hline $4^{\circ}$ & AUTOSUFICIENTE & $1^{\circ}$ \\
\hline & SENTIMENTAL & $5^{\circ}$ \\
\hline
\end{tabular}

Elaboración propia 
Tabla $\mathrm{n} \div$ 4. Resultados de la segunda TGN con alumnas y alumnos, sobre lo masculino y su importancia comparada con la que se le había otorgado en la 1a TGN

\begin{tabular}{|c|c|c|}
\hline $\begin{array}{c}\text { ORDEN SEGÚN LA } \\
\text { PUNTUACIÓN TGN } \\
\text { ANTERIOR }\end{array}$ & $\begin{array}{c}\text { ORDEN SEGÚN LA } \\
\text { IMPORTANCIA EN ESTA } \\
\text { TGN }\end{array}$ \\
\hline $1^{\circ}$ & HONRINO UTILIZADO & $2^{\circ}$ \\
\hline $2^{\circ}$ & TRABAJADOR & $1^{\circ}$ \\
\hline $4^{\circ}$ & FUERTE & $4^{\circ}$ \\
\hline $5^{\circ}$ & SENSIBLE & $5^{\circ}$ \\
\hline $6^{\circ}$ & TOLERANTE & $3^{\circ}$ \\
\hline
\end{tabular}

Elaboración propia

Tabla nำ 5. Resultados de la importancia del perfil masculino y su variación según se hiciera el análisis por cada uno de los géneros o conjuntamente

\begin{tabular}{|c|c|c|c|c|}
\hline \multirow{2}{*}{$\begin{array}{c}\text { ORDEN DE } \\
\text { IMPORTANCIA }\end{array}$} & \multicolumn{2}{|c|}{ FEMENINO } & \multicolumn{2}{c|}{ MASCULINO } \\
\hline 1 & ALUMNAS & ALUMNAS Y ALUMNOS & ALUMNOS & ALUMNOS Y ALUMNAS \\
\hline 2 & LUCHADORA & INDEPENDIENTE & HONRADO & TRABAJADOR \\
\hline 3 & UNNICA & LUCHADORA & LEAL & TOLERANTE \\
\hline 4 & LIBERAL & ÚNICA & FUERTE & FUERTE \\
\hline 5 & AUTOSUFICIENTE & SENTIMENTAL & SENSIBLE & SENSIBLE \\
\hline 6 & PERSONA & & TOLERANTE & \\
\hline
\end{tabular}

Elaboración propia

\begin{tabular}{|c|c|c|}
\hline CONCEPTO & DICCIONARIO & OPINIÓN DE LAS ALUMNAS \\
\hline Independiente & $\begin{array}{l}\text { Que sostiene sus derechos u opiniones } \\
\text { sin admititi intervención ajena }\end{array}$ & $\begin{array}{l}\text { No depender ni económica ni emocionalmente de } \\
\text { nadie }\end{array}$ \\
\hline Luchadora & $\begin{array}{l}\text { Tenaz en el esfuerzo para sacar adelante } \\
\text { su propósito }\end{array}$ & Va en pos de sus objetivos personales \\
\hline Única & Sólo y sin otro de su especie & $\begin{array}{l}\text { Un ser absolutamente individual y deferente a } \\
\text { las/los demás }\end{array}$ \\
\hline Liberal & Inclinada a la libertad, comprensiva & $\begin{array}{l}\text { Respetuosa con las ideas, creencias o prácticas de } \\
\text { llos demás, pero con una actitud de poder elegir } \\
\end{array}$ \\
\hline Autosuficiente & Que se basta a si misma & $\begin{array}{l}\text { Cuyo valor se debe a si misma y necesita menos de } \\
\text { los demás }\end{array}$ \\
\hline Persona & $\begin{array}{l}\text { Mujer de prendas, capacidad, } \\
\text { disposición y prudencia }\end{array}$ & $\begin{array}{l}\text { Evitar ser encasillada en una categoria (por ejemplo } \\
\text { el género) }\end{array}$ \\
\hline Sentimental & $\begin{array}{l}\text { Propensa a albergar o suscitar } \\
\text { sentimientos tiernos o amorosos }\end{array}$ & Actúan y reaccionan emotivamente \\
\hline
\end{tabular}

Tabla $n^{\circ} 7$. Comparación de los términos que definen lo masculino con definiciones del Diccionario de la Lengua

\begin{tabular}{|c|l|l|}
\hline CONCEPTO & \multicolumn{1}{|c|}{ DICCIONARIO } & \multicolumn{1}{|c|}{ OPINIÓN DE LOS ALUMNOS } \\
\hline Honrado & $\begin{array}{l}\text { Que procede con rectitud de ánimo e } \\
\text { integridad en el obrar }\end{array}$ & Que se puede confiar en él, y él en los demás \\
\hline Trabajador & Muy aplicado al trabajo & $\begin{array}{l}\text { Alguien que se esfuerza por subir y mejorar su } \\
\text { estatus laboral y vital }\end{array}$ \\
\hline Leal & $\begin{array}{l}\text { Que guarda a alguien o a algo la debida } \\
\text { fidelidad }\end{array}$ & $\begin{array}{l}\text { Un hombre siempre está ahí, de forma humilde } \\
\text { y veraz }\end{array}$ \\
\hline Fuerte & De carácter firme y animoso & Fuerte psiquicamente \\
\hline Sensible & Que siente fisica y moralmente & $\begin{array}{l}\text { La sensibilidad es inherente al hombre, y no } \\
\text { puede el esterotipo de macho impedirlo }\end{array}$ \\
\hline Tolerante & $\begin{array}{l}\text { Respeta las ideas, creencias o prácticas } \\
\text { de los demás cuando son diferentes o } \\
\text { contrarias a las propias }\end{array}$ & $\begin{array}{l}\text { Abierto de ideas, capaz de compartirlas e } \\
\text { intercambiarlas, y cambiar de opinión }\end{array}$ \\
\hline
\end{tabular}

